\title{
DNA copy number, including telomeres and mitochondria, assayed using next-generation sequencing
}

\author{
John C Castle*1,2, Matthew Biery1,3, Heather Bouzek1,4, Tao Xie 1,5, Ronghua Chen 1,6, Kira Misura1,7, Stuart Jackson 1,6, \\ Christopher D Armour1,3, Jason M Johnson 1,6, Carol A Rohl1,6 and Christopher K Raymond*1,3
}

\begin{abstract}
Background: DNA copy number variations occur within populations and aberrations can cause disease. We sought to develop an improved lab-automatable, cost-efficient, accurate platform to profile DNA copy number.

Results: We developed a sequencing-based assay of nuclear, mitochondrial, and telomeric DNA copy number that draws on the unbiased nature of next-generation sequencing and incorporates techniques developed for RNA expression profiling. To demonstrate this platform, we assayed UMC-11 cells using 5 million $33 \mathrm{nt}$ reads and found tremendous copy number variation, including regions of single and homogeneous deletions and amplifications to 29 copies; 5 times more mitochondria and 4 times less telomeric sequence than a pool of non-diseased, blood-derived DNA; and that UMC-11 was derived from a male individual.

Conclusion: The described assay outputs absolute copy number, outputs an error estimate ( $p$-value), and is more accurate than array-based platforms at high copy number. The platform enables profiling of mitochondrial levels and telomeric length. The assay is lab-automatable and has a genomic resolution and cost that are tunable based on the number of sequence reads.
\end{abstract}

\section{Background}

DNA copy number variations occur within populations and aberrations can cause tumors, be used for drug target identification, and be used as biomarkers of tumor drug response. EGFR (epidermal growth factor receptor) amplification, for instance, is a marker for gefitinib treatment [1] and TYMS (thymidylate synthase) amplification conveys 5-fluorouracil resistance in colon tumors [2].

The next-generation sequence enables generation of millions of short sequence tags in a single experiment. Using DNA as an input, the technology has been used to resequence entire genomes, including from normal indi-

* Correspondence: castle@uni-mainz.de

, raymondsall@comcast.net

1 Rosetta Inpharmatics LLC, a wholly owned subsidiary of Merck \& Co., Inc., Seattle, Washington 98109, USA

1 Rosetta Inpharmatics LLC, a wholly owned subsidiary of Merck \& Co., Inc., Seattle, Washington 98109, USA

Full list of author information is available at the end of the article viduals [3] and from cancerous cells [4], and to resequence targeted genomic regions, such as resequencing protein coding regions to discover somatic mutations [5]. Alternatively, using RNA as an input, the technology has been used to profile RNA expression levels, where the number of sequence reads "tagging" an RNA transcript is a measure of its expression [6].

Combining these ideas and building on previous methods [7-10], this report describes the development of a sequencing-based platform to profile "expression" of DNA, inputting DNA and analyzing the resultant data using algorithms previously established for gene expression profiling. The platform resolution and cost are tunable; is lab automatable; can be used for both discovery of novel and profiling of known DNA copy number variations; outputs copy number and uncertainty as opposed to ratios; and can be used to profile mitochondria and telomeric DNA. 


\section{Results}

Application 1: Assaying nuclear genomic copy number We assayed the genomic copy number in a pool of DNA derived from blood from non-diseased males; a pool of DNA derived from blood from non-diseased females; and DNA from UMC-11 cells, a lung carcinoid-derived cell line. We generated and sequenced each library, aligned resultant reads to the genome, and selected reads aligning to only one genomic location (Methods).

Across chromosome 9, the number of reads from the male pool mapping to each genomic block is near 250 (Figure 1A, blue). From the female pool, the number of reads mapping to each block is similarly constant, at roughly 150 (Figure 1A, green). We tested normalization by the GC content in each DNA block $[9,10]$; however, we found that the relative number of reads mapping to a block depended more strongly on molecular biology protocols. Instead, we developed and applied a novel normalization method using the male pool as a reference, and with this normalization were able to derive the copy number, upper and lower bounds, and the significance of any deviation (Methods). In the female DNA pool, the copy number is two across the entire chromosome, with a single exception at position $\sim 95 \mathrm{Mb}$ (Figure 1B). Use of a single reference sample, processed using the same bench protocol as used for the non-reference samples, therefore
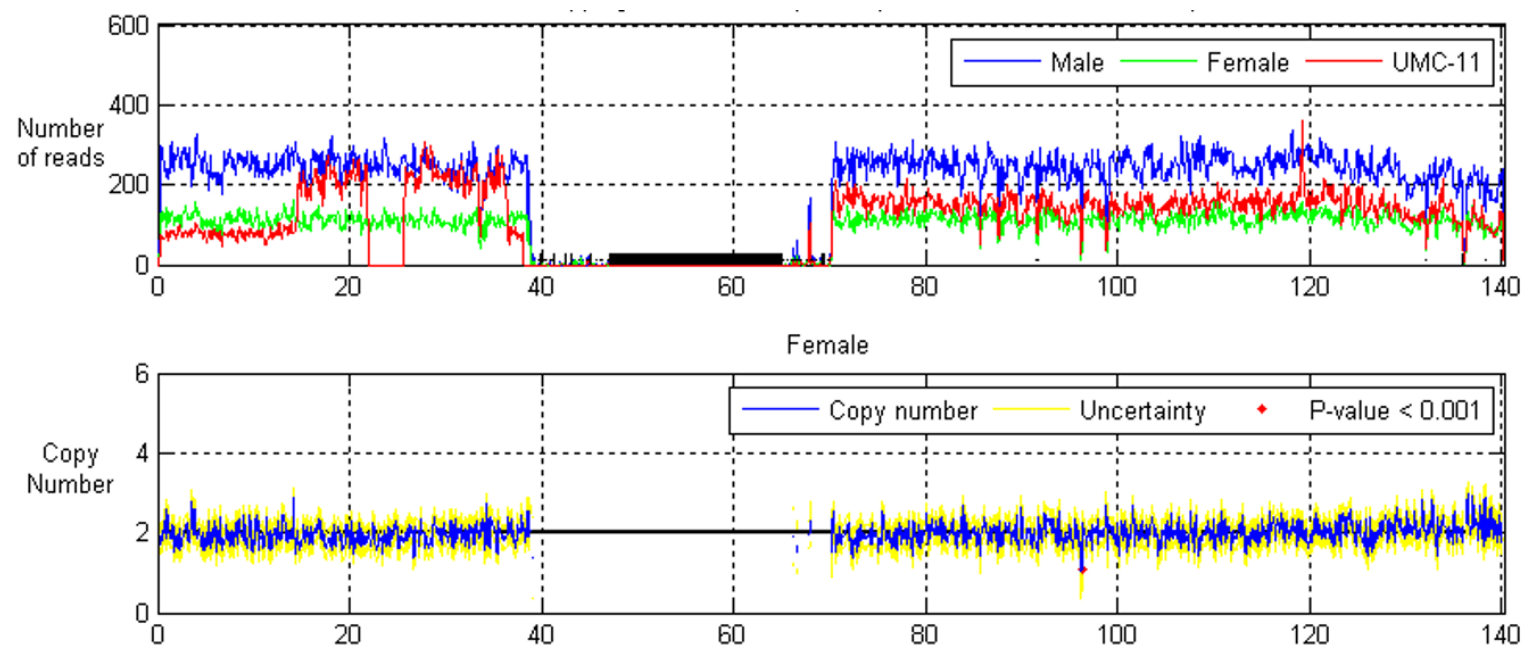

UMC-11



Figure 1 Copy number across chromosome $\mathbf{9}$ in the male pool, female pool and UMC-11 cells. Top: The number of sequence reads mapping to $150 \mathrm{~kb}$ windows, stepping at $75 \mathrm{~kb}$. Second: Copy number in the female pool. Blue line, copy number; yellow lines, upper and lower bounds; red points, windows with copy number variation ( $p$-value < 0.001). Third: Copy number in the UMC-11 cells. Bottom: Transcripts/genes in the deleted region. 
eliminates the need to normalize for GC content and for sequence uniqueness within each block.

Conversely, UMC-11 cells show dramatic copy number variations. Before normalization, the number of reads mapping to each block shows many discrete levels, including blocks with no reads and blocks with roughly 75, 150, and 225 reads (Figure 1A, red). After normalization, these discrete levels map to copy number of zero, one, two, three, and four (Figure 1C). Intriguingly, the homozygously deleted region spanning from 21 to $24 \mathrm{Mb}$ includes the putative tumor suppressor CDKN2A [11] (Figure 1D).

Across chromosome 12, the female pool has copy number two across the entire chromosome, as expected (Figure $2 \mathrm{~B}$ ). The UMC-11 cell line is highly aberrant: copy number starts at three; is amplified to 9; falls to three; is amplified up to 29 copies; drops to three; falls to two; has a small segment at three copies; back to two; and then back to three. Again intriguingly, the highly amplified region includes the tumor-associated gene KRAS [12].

This assay provides a genome-wide view of copy number in a single assay, allowing the full extent of copy number within a sample to be assessed (Figure 3). While chromosomes $13,16,21,22$, and X in UMC-11 cells show little disruption, many chromosomes show amazing copy number variation. Almost $50 \mathrm{Mb}$ of chromosome 5 is present at 5 copies. An $80 \mathrm{Mb}$ block of chromosome 6 is present at one copy. Many changes in copy number occur close to or across centromeres. We also identify that the cell line is derived from a male patient as chromosome $\mathrm{X}$ is present at one copy and many reads map to chromosome Y.

To validate findings, we assayed copy number with qPCR at 12 locations (Figure 3, blue boxes; Table 1, Methods). The five locations predicted to have copy number two by sequencing indeed had copy number two as measured by qPCR. Copy number measurements of $1,3,4$, and 12 also validated. At high copy number, sequencing and qPCR measurements were similar: 10 and 11; 26 and 24; 25 and 29, respectively. These results also show high correspondence with findings from an array-based platform [7] (Table 1, Additional files 1 and 2: Figures S1 and S2), including the chromosome 9 deletion near $22 \mathrm{Mb}$ and the small amplification near position $120 \mathrm{Mb}$. Results from the highly amplified region of chromosome 12 qualitatively agree; however, the array-based method predicts only 13 copy number in the region where the sequencing and qPCR assays measure over 25 copy number (Table 1 ). Across the entire genome, the array-based data for UMC11 cells, downloaded from the Sanger Center and analyzed with the PICNIC algorithm [13], show high concordance at copy number five and below, but discrepancies at higher copy number (Figure 4). The array-platform returns lower copy numbers than found by $\mathrm{qPCR}$ and sequencing, potentially the result of microarray probe saturation [8].

\section{Application 2: Assaying genetic loci copy number}

Conceptually, one can define biological elements in terms of gene loci rather than genomic blocks, and thus, by counting the number of reads uniquely mapping to each locus, generate a gene-copy number table. Here, we defined locus coordinates as the greater of the transcript start-to-stop span or $60 \mathrm{~kb}$ centered on the loci. As before, we normalized the counts to the reference male pool, assuming the male pool is diploid across autosomes and haploid across allosomes.

The loci with the most significant p-values for higher copy number in the female pool are, not surprisingly, found on chromosome X (Figure 5A). In each case, this platform assayed the loci at copy number two in the female pool and one in the male pool and UMC-11 cells. The loci with the most significant lower copy number in UMC-11 cells are in the chromosome 9 deletion (Figure $5 \mathrm{~B})$, where the loci show copy number two in the female pool. The loci with the most significant amplifications are in the chromosome 12 amplification (Figure 5C), with copy number at almost 30.

Similarly, biological elements can be defined in terms of the coordinates of known DNA copy number polymorphisms [14]. By counting reads aligning within the coordinates of each established polymorphism, one can monitor population-variable copy number polymorphisms (not shown).

\section{Application 3: Mitochondrial copy number}

Biological elements can also be defined as the mitochondrial DNA (mtDNA), in which case one assays the number of mitochondria in the cells. While the counts of mtDNA within each individual cell will vary, bloodderived cells on average contain fewer mitochondria relative to cell lines [15]. From the aligned sequence reads, we counted and normalized the number of reads aligning to mtDNA as a measure of the average mtDNA levels within each sample. Indeed, we found that the UMC-11 cells contain over 5 times more mitochondria than the bloodderived male and female pools (Figure 6A).

\section{Application 4: Telomere copy number}

Finally, another fascinating biological element that can be interrogated is telomeric sequence. Telomeres protect the ends of chromosomes; are on average shorter in cells that have undergone many divisions, such as older cells, tumors, and cell lines; and comprise repetitive TTAGGG motifs [16]. We counted and normalized the number of sequence reads containing (TTAGGG) ${ }_{4}$. Strikingly, the UMC-11 cells do contain significantly fewer telomere- 

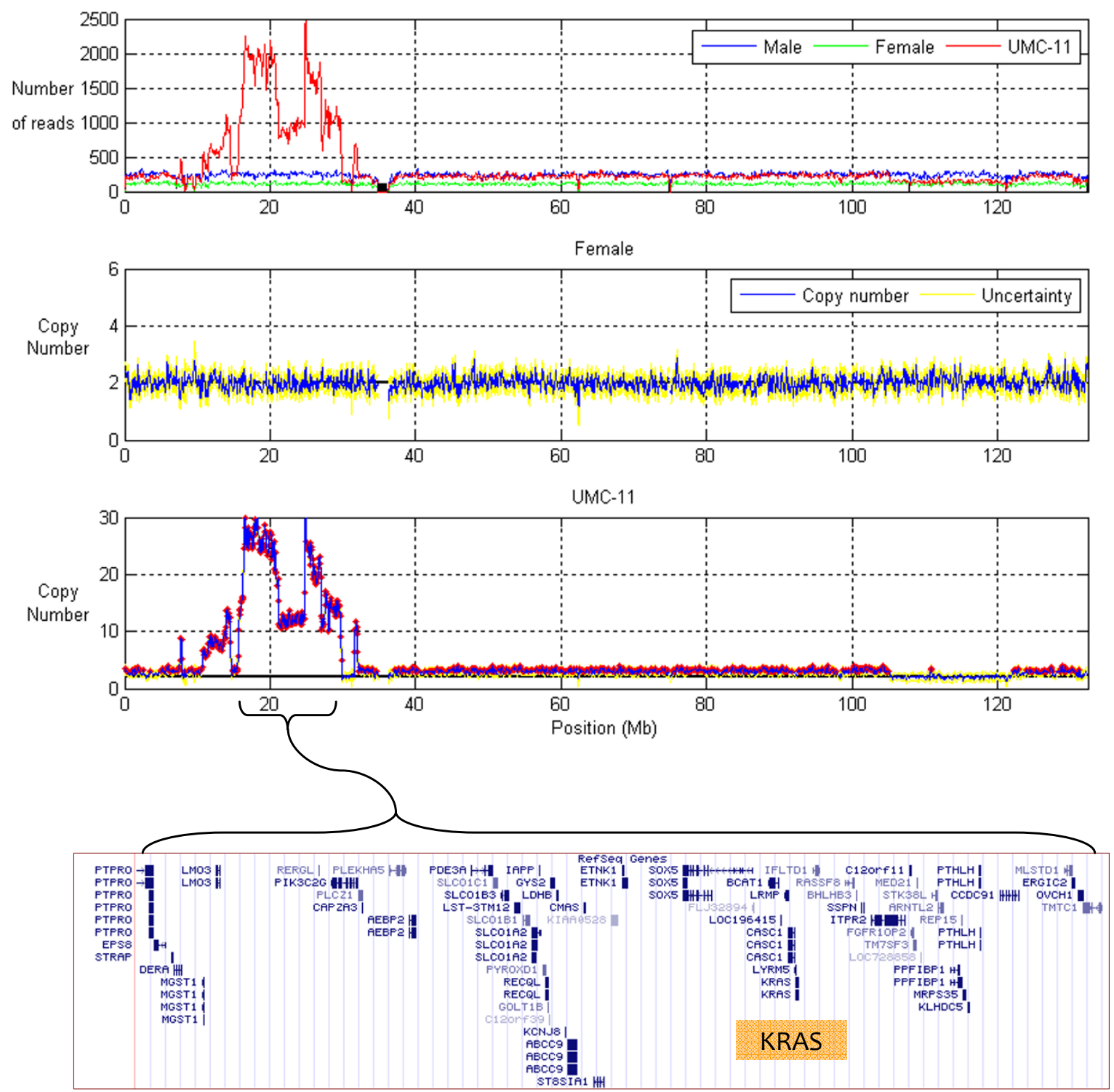

Figure 2 Copy number across chromosome 12, as per Figure 1.

associated reads than either the female or male pools (Figure 6B).

\section{Discussion and Conclusions}

Assaying DNA copy number by next-generation sequencing is robust and accurate. The method described here requires a simple genomic DNA library construction; returns integer copy number values for homogeneous cells; and has a large dynamic range. The platform is unbiased in the sense that genomic targets are not preselected, such as is the case with qPCR and microarrays, and thus, given a new genome assembly, a new set of copy number polymorphisms, or a new set of biological DNA elements, the sequence reads maintain utility through realignment.

Our research builds on previous efforts. The 'digital karyotyping' protocol uses restriction enzymes and SAGE sequencing technology to generate reads that have been used to measure copy number variation and detect infectious viral DNA[17,18]. Using Illumina deep-sequencing, fetal aneuploidy was assayed, identifying Down, Edward, and Patau syndromes based on chromosome-specific trisomy [19]. Illumina deep-sequencing has been used to examine copy number across nuclear chromosomes. 

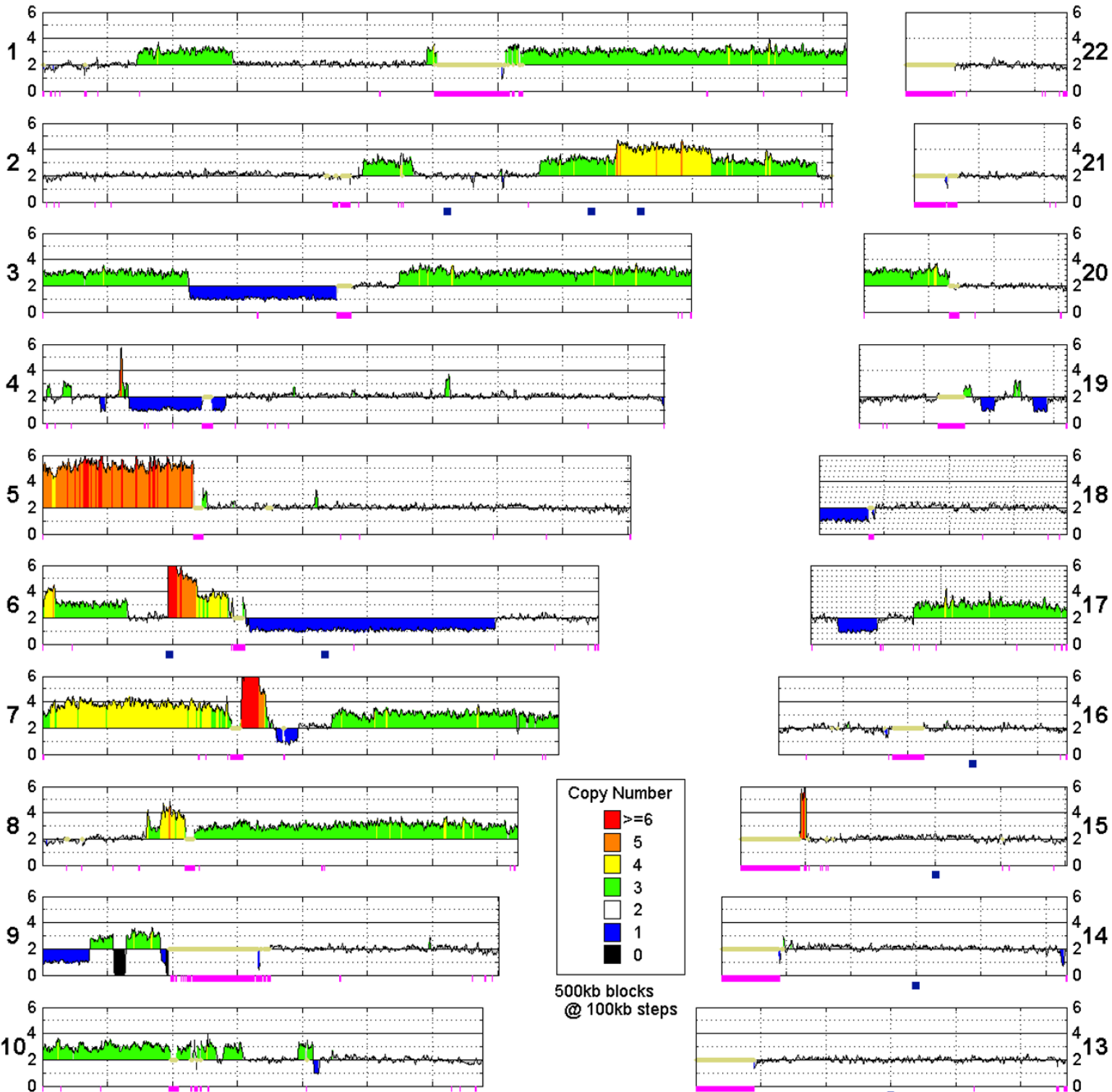

$500 \mathrm{~kb}$ blocks (100kb steps
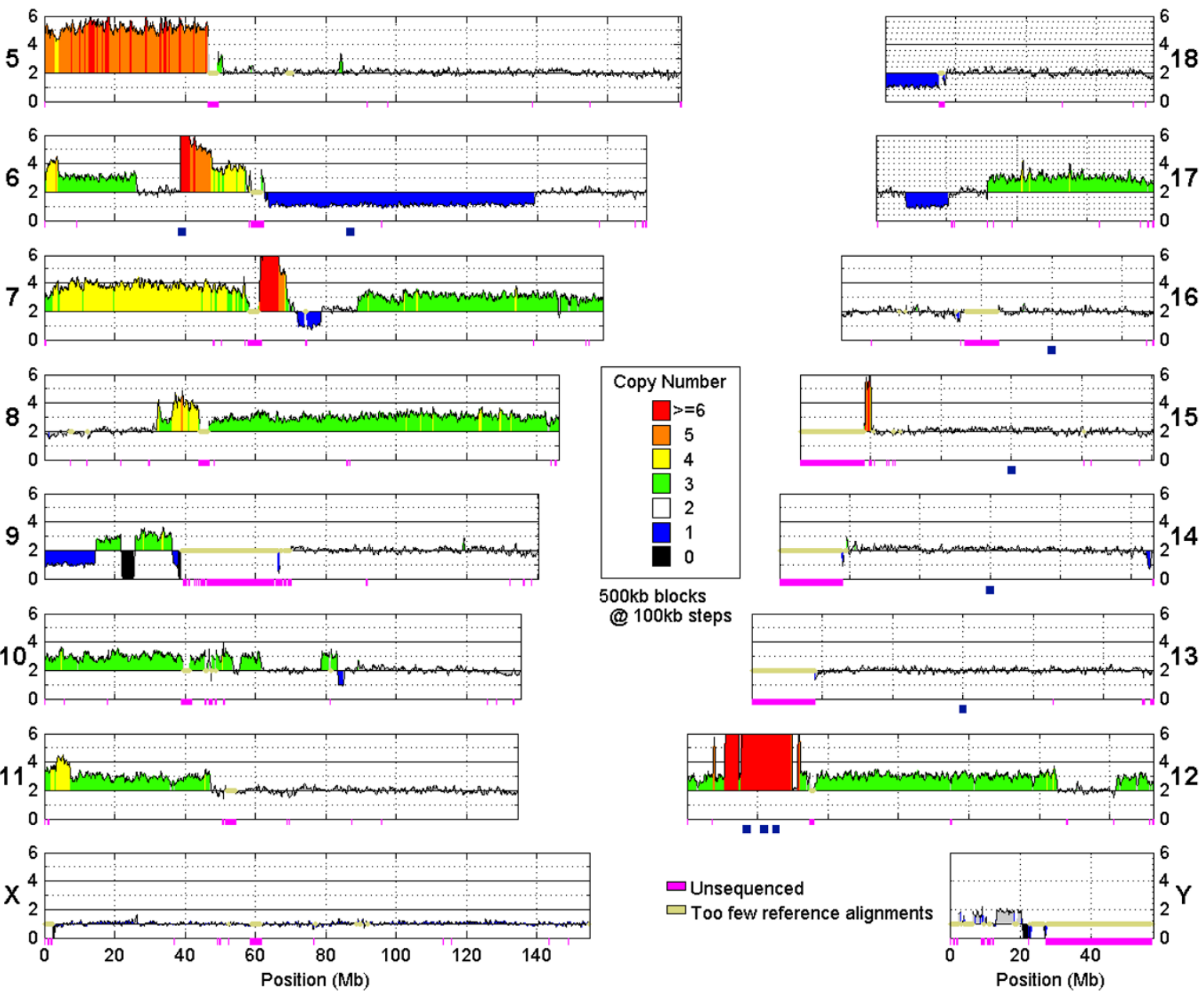

Figure 3 Genome-wide copy number in the UMC-11 cells. Copy number is assayed in 500 kb windows, stepping at $100 \mathrm{~kb}$. Blocks are assigned color based on the nearest integer. Tan regions contain fewer than 50 uniquely aligning reads in the reference sample. Below each chromosome, pink marks unsequenced regions and the 12 blue boxes mark the locations assayed by qPCR. 


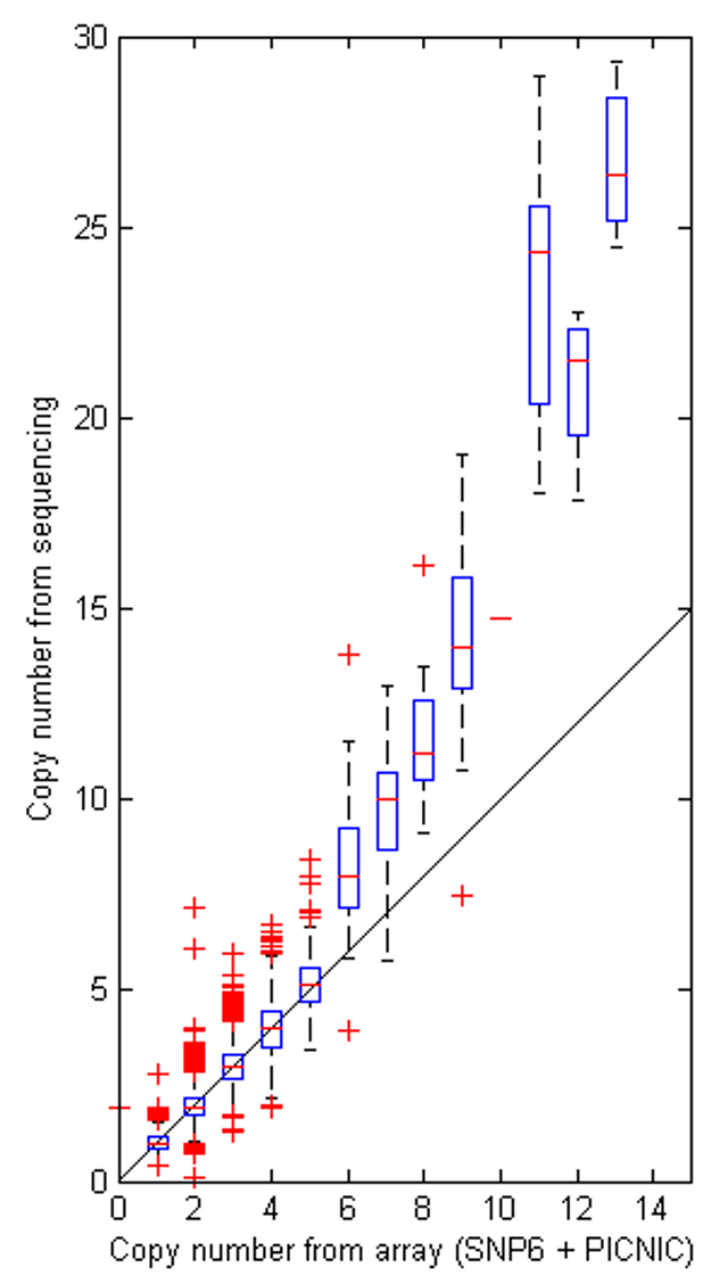

Figure 4 Comparison between UMC-11 copy number from sequencing platform with that from Affymetrix SNP6 arrays analyzed using the Sanger PICNIC algorithm.

Campbell et al[7] used paired-end reads from sizeselected libraries to identify genomic structural rearrangements and, integrating estimates of copy number with paired-end reads mapping to distal locations, were able to identify breakpoint coordinates and novel DNA sutures. Recently, Chiang et al[8] sequenced a sizeselected library and measured the log-ratio change between normal and tumor sample pairs across nuclear chromosomes. They elegantly show trade-off curves between read number, copy number change, and genomic resolution and show statistical determination of breakpoints. In comparing their results to array-based methods, they find the sequencing-based platform has a larger dynamic range. Yoon et al. [10] and Alkan et al. [9] developed similar methods for use in human resequencing projects, using over 1 billion reads and $30 \times$ genome cov- erage to identify copy number polymorphisms in diseasefree cells.

The method described here expands on these groundbreaking studies in several ways. First, our library construction does not include a size selection and is thus lab automatable. Second, by using a single diploid reference sample along with a novel normalization algorithm, our method removes biases inherent in molecular biology protocols and outputs absolute copy number in addition to $\log$-ratio values. Third, we defined an uncertainty that allows us to estimate upper and lower bounds and p-values for each copy number measurement, both for absolute and relative measurements. Forth, by defining biological elements as not only nuclear DNA blocks positioned evenly across the nuclear genome, this platform enables assaying other biologically meaningful DNA elements, including gene loci, known copy number polymorphisms, mitochondria, and telomeres.

Finally, these results were generated using an Illumina Genome Analyzer II instrument in June, 2008, with one sample per lane, resulting in only 3 to 7 million $36 \mathrm{nt}$ reads per sample. Sequencing instrumentation continues to improve, allowing more reads at lower costs. As the resolution of this assay is inversely proportional to the number of aligned reads, sample multiplexing and increasing numbers of sequence reads will enable increased resolution and/or significantly decreased costs.

\section{Methods}

\section{Proof of concept of the sequencing-based DNA copy} number profiling assay

To develop and evaluate a sequencing based platform for assaying CNV, we prepared genomic DNA libraries from a pool of DNA derived from blood from non-diseased males; a pool of DNA derived from blood from non-diseased females; and DNA from the UMC-11 cell line, a lung carcinoid-derived cell line. DNA was fragmented using DNase. We did not test whether DNase can shear heterochromatin sequence such as in centromeres; however, we used only sequence reads that align uniquely to the genome and thus would not use sequence reads from regions of low sequence-complexity. The ends of the fragmented DNA were filled in or cleaved to produce blunt ends. This blunted DNA was ligated to adapters containing reverse complements on like adapters for suppression PCR as well as priming sites for a set of universal PCR primers and the Illumina sequencing primer. These "DNA Libraries" were then amplified using standard PCR conditions. We did not use size-selection after fragmentation, allowing lab-automation of library construction.

We sequenced libraries in the Illumina Genome Analyzer II sequencer, generating 6,744,152 (male pool), $3,283,370$ (female pool), and 5,204,934 (UMC-11) reads 
Table 1: Validation of sequencing copy number assayed by qPCR.

\begin{tabular}{ccccc}
\hline Chromosome & Coordinate & qPCR & Sequencing & Array \\
\hline 2 & $124,585,349$ & 2 & 2 & 2 \\
2 & $168,899,639$ & 3 & 3 & 3 \\
2 & $184,059,790$ & 4 & 10 & 7 \\
6 & $39,100,000$ & 11 & 1 & 1 \\
6 & $87,009,800$ & 1 & 26 & 13 \\
12 & $16,995,694$ & 24 & 12 \\
12 & $21,762,192$ & 12 & 25 & 11 \\
12 & $25,206,027$ & 29 & 2 & 2 \\
13 & $60,000,100$ & 2 & 2 & 2 \\
15 & $60,000,100$ & 2 & 2 & 2 \\
\hline
\end{tabular}

Values are rounded to the nearest integer.

(Table 2) (NCBI GEO accession GSE21159) Each $36 \mathrm{nt}$ read contained a $3 \mathrm{nt}$ molecular barcode for potential sample multiplexing which was trimmed before alignment.

\section{Genomic alignment}

The resultant sequence reads were computationally aligned to the genome using the algorithm BWA [20]. We used only reads aligning to the genome with the highest (least ambiguous) score, minimizing incorrect mappings such as those from the female pool mapping to the $\mathrm{Y}$ chromosome. A shortcoming of the use of uniquely aligning reads is that reads aligning to sequence-identical segmental duplications within the HG18 genome will be discarded and these regions not monitored. Between 92\% and $93 \%$ of the reads aligned to the genome, with between $63 \%$ and $65 \%$ aligning unambiguously (Table 2). For each unambiguously aligned read, we recorded the chromosome and 5 ' coordinate.

We computationally tested use of different read lengths. Shorter reads are more likely to align ambiguously whereas longer reads are more likely to align to a unique genomic location. We computationally generated all oligos (tiles) from the human genome at lengths from $20 \mathrm{nt}$ to $200 \mathrm{nt}$. We aligned all to the genome and, for each tile length, determined the fraction of the reads that aligned to only one location (Figure 7). Seven percent of the human genome remains undetermined, containing repeat and low-complexity sequence patterns, e.g. centromeres.

Only $26 \%$ of the $20 \mathrm{nt}$ tiles align uniquely, increasing to $65 \%$ at $25 \mathrm{nt}$, and to $70 \%$ at $30 \mathrm{nt}$, and smoothly increasing to $90 \%$ at $200 \mathrm{nt}$. A fundamental shift occurs between 20 and 25 nt: genome uniqueness changes from majority ambiguous to majority unambiguous. The 33 nt length (36 nt minus the 3 nt barcode) used in the study here represents an effective trade-off between sequencing costs (time and money) and read uniqueness.

\section{Counting \& uncertainty}

We selected biological elements for examination, such as DNA windows. For each window, we counted the number of reads aligning within the window. We compared the number to that found in a diploid reference sample. We used the DNA pooled from non-diseased males as a reference as the DNA should be diploid across autosomes and represent all chromosomes, including chromosome Y.

We defined an error model for a measurement " $x$ " as a Poisson term from counting statistics:

$$
\sigma=\sqrt{x}
$$

With this error model, a block with 100 reads would have an uncertainty of \pm 10 . This error model reflects sampling uncertainty but neither biological or operator variability.

We used a uniform window size across the genome; however, a variable window size could be selected. The selected window size should reflect the total number of uniquely aligned reads available, the desired sensitivity/ significance, and the sample cell-to-cell genomic heterogeneity. Here, we assumed that the cellular DNA was homogeneous and wished to have the power to distinguish copy number 3 from 2 at a p-value better than 0.001 assuming the described error model. This equates to 110 reads per genomic block. As we had 3.3 million reads from the female blood pool, of which 2.1 million uniquely aligned to the genome, and given the human genome of 

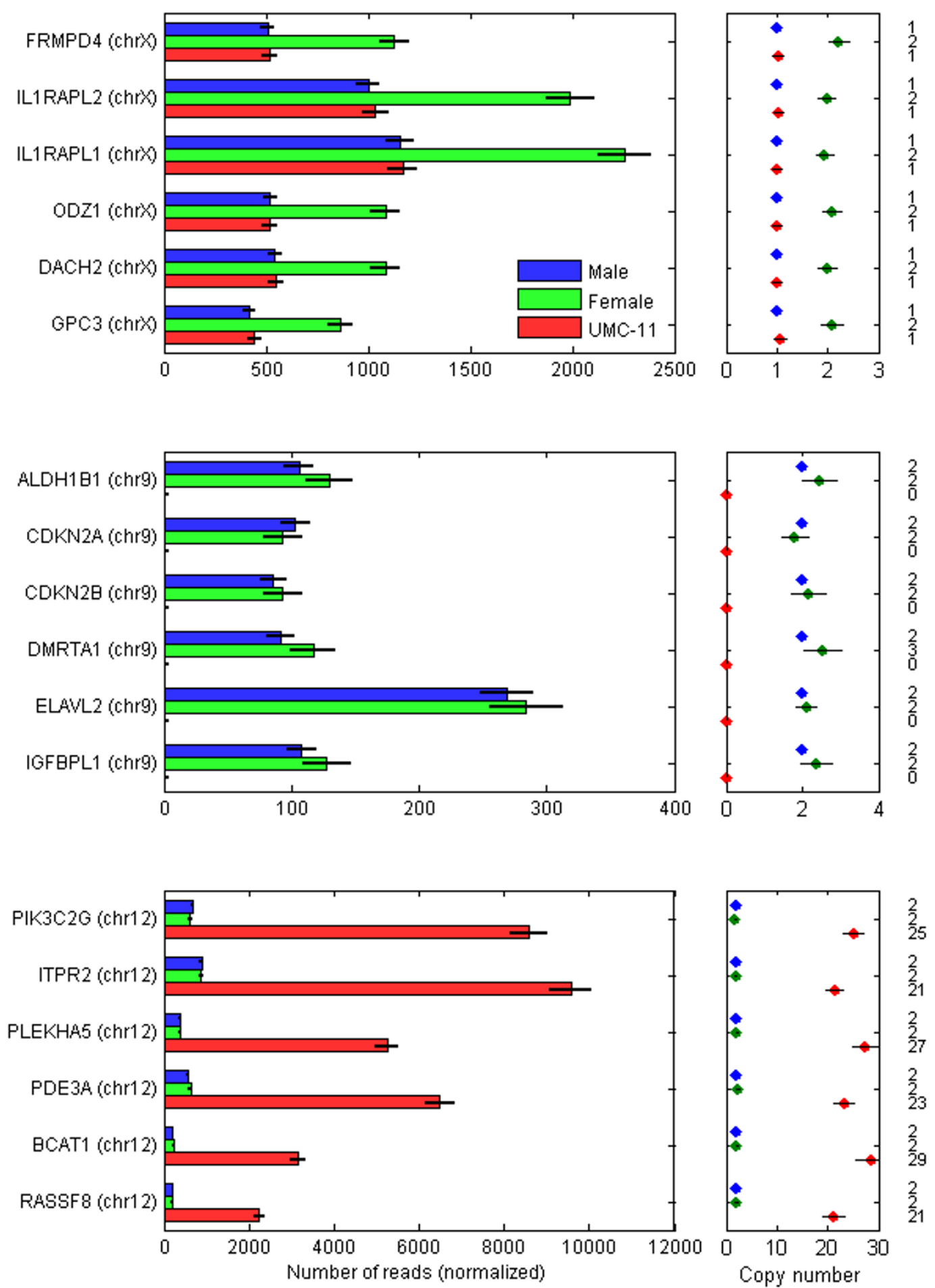

Figure 5 Gene-centric copy number. Top: the six genes with the most significant amplification in the female pool. Middle: the six genes with the most significant decreased copy number in the UMC-11 cells. Bottom: the six genes with the most significant amplification in the UMC-11 cells. The male pool is assumed to be diploid (autosomes) and haploid (allosomes). 


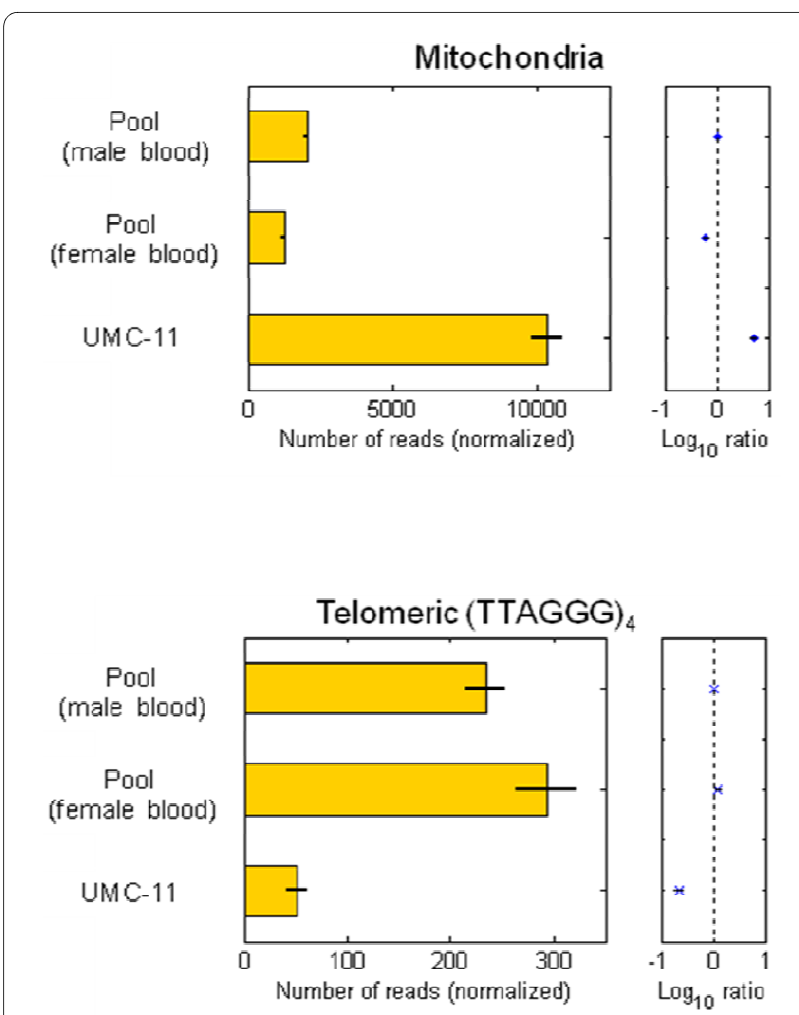

Figure 6 Mitochondria and telomere copy number.

3.1 billion base pairs, this results in a block size of $164 \mathrm{~kb}$. We rounded to $150 \mathrm{~kb}$ blocks.

\section{Normalization}

We normalized reads to account for the differing numbers of reads per sample and to determine absolute copy number. We found that the relative number of reads mapping to a DNA block depended on the molecular biology protocol used, thus necessitating use of a empirical normalization rather than simply a measure such as GC content. We used a male pool as a reference, and with this normalization were able to derive the copy number, upper and lower bounds, and the significance of any variation.

We counted the reads mapping to each $150 \mathrm{~kb}$ block from the male and female pools and generated a ratio, female-to-male, for each block. There were roughly twice the number of reads for the male sample (Table 1), and

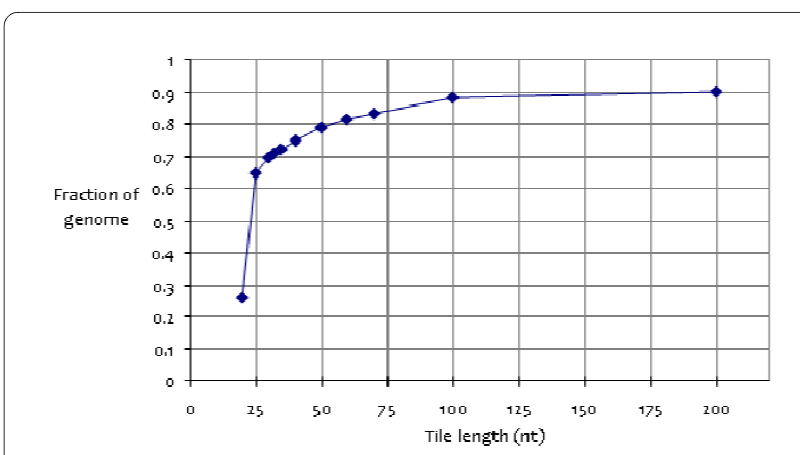

Figure $\mathbf{7}$ Genome uniqueness at small scale lengths. At each length scale, all oligos were aligned to the genome and unambiguously aligned reads were counted. The $y$-axis includes unsequenced regions (7\%).

thus the distribution of ratios peaks near 0.5 (Figure 8, top left). Normalizing by the number of aligned reads from the female and male samples results in a distribution peaked near one, as expected for samples with similar ploidy, and copy number two, assuming the male sample pool is diploid across autosomes (Figure 8, lower left).

The distribution of UMC-11 to male ratios after normalization by the number of reads shows multiple peaks, none of which are centered at an integer (Figure 8, middle right). We tried using the ratios to determine the normalization: using the ratio mean or the median failed to center a distribution peak at an integer. However, we were able to normalize the counts based on the mode of the ratio distribution across autosomes. After normalization by the ratio model, we find that the three peaks in the ratio distribution from the UMC-11 cell line are regions with one, two, and three copy number (Figure 8, lower right).

\section{Comparisons between samples}

To compare CNV between samples, we calculated the significance of a difference using a t-test and p-value based on the normalized counts and normalized uncertainties. For each DNA element, the assay thus returns the absolute copy number, uncertainty, upper and lower bounds, and a p-value representing the significance of a measured difference.

Table 2: Sequencing reads ( $33 \mathrm{nt}$ ) and alignments.

\begin{tabular}{|c|c|c|c|c|c|}
\hline Sample & Reads & $\begin{array}{l}\text { Aligned to } \\
\text { genome }\end{array}$ & $\%$ & $\begin{array}{c}\text { Unambiguous } \\
\text { match }\end{array}$ & $\%$ \\
\hline Female pool & $3,283,370$ & $3,038,662$ & $93 \%$ & $2,061,012$ & $63 \%$ \\
\hline Male pool & $6,744,152$ & $6,193,928$ & $92 \%$ & $4,372,811$ & $65 \%$ \\
\hline UMC-11 & $5,204,934$ & $4,768,159$ & $92 \%$ & $3,313,093$ & $64 \%$ \\
\hline
\end{tabular}




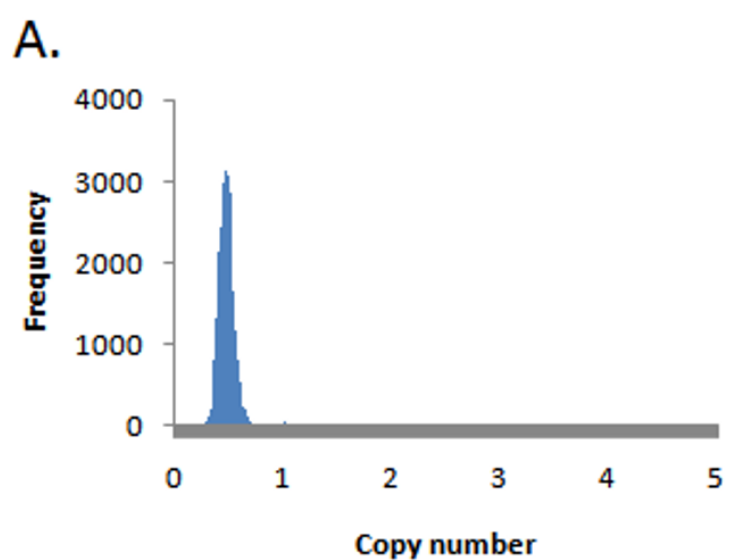

B.

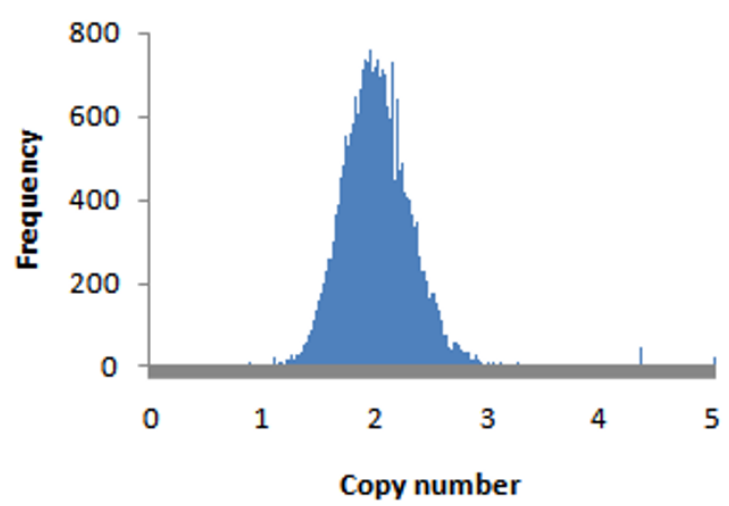

C.

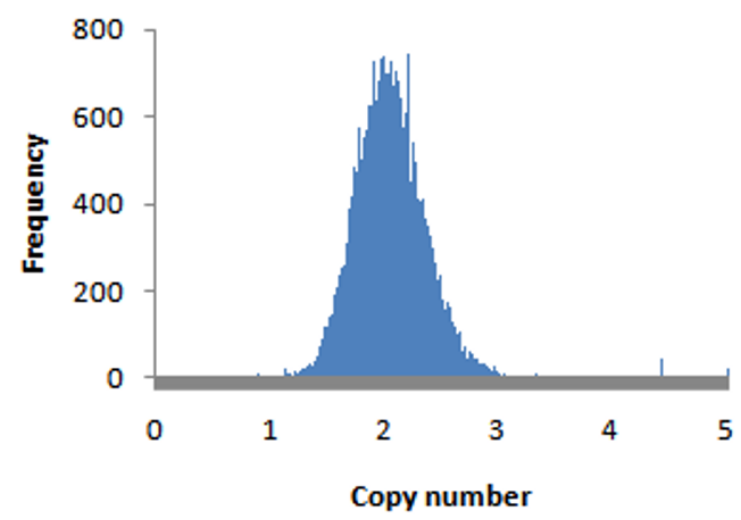

D.

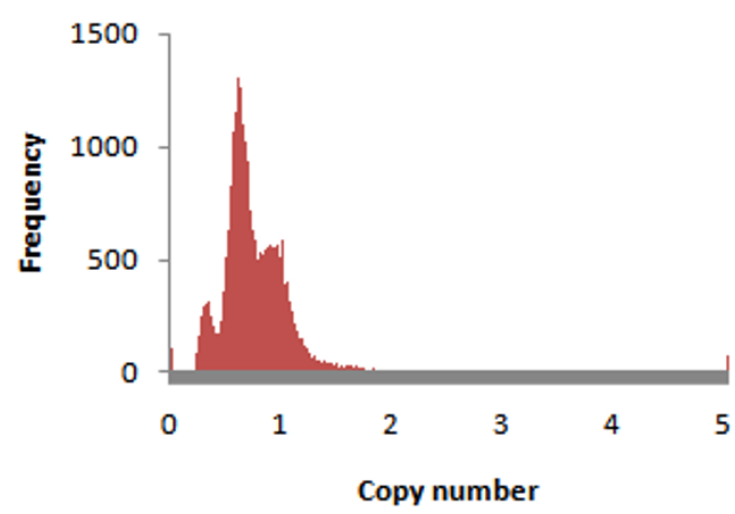

E.

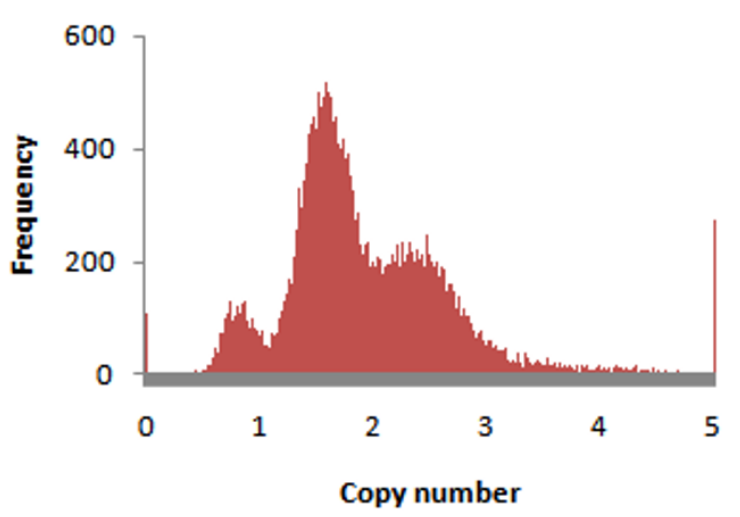

F.

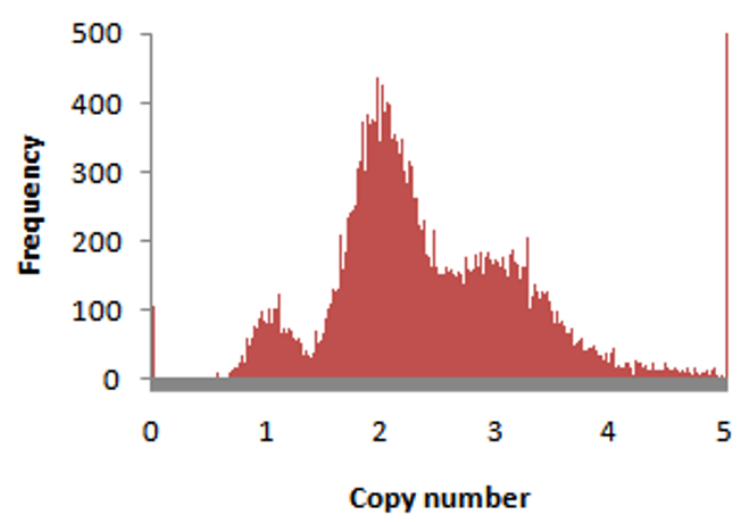

Figure 8 Normalization allows absolute copy number estimate after normalization, assuming the male pool is diploid across all autosomes. Left: female pool (blue). Right: UMC-11 (red). A and D: genome-wide distribution of the ratios of the number of reads aligning in each $150 \mathrm{~kb}$ window relative to the number in the same window from the male pool. B and $\mathrm{E}$ : counts are normalized by the total number of unambiguously aligned reads from each sample. C and F: counts are normalized by the mode from the distribution in B and E. Copy number of 1, 2, and 3 can be clearly seen in the UMC-11 sample. 


\section{Validation with qPCR}

We used qPCR (Taqman) to assay DNA copy number in UMC-11 cells. TaqMan primer-probe reagents were obtained through the Applied Biosystems Assays-byDesign custom assay service (Foster City, CA) and designed to fall outside of repeat regions.

\section{Additional material}

Additional file 1 Figure S1. UMC-11 chromosome 9 copy number from the deep-sequencing platform (top) and Affymetrix SNP6 arrays and the Sanger Picnic algorithm [13] (bottom, green line).

Additional file 2 Figure S2. UMC-11 chromosome 12 copy number from the deep-sequencing platform (top) and Affymetrix SNP6 arrays with the Sanger Picnic algorithm [13] (bottom, green line).

\section{Competing interests}

The authors declare that they have no competing interests.

\section{Authors' contributions}

JCC and CKR designed the experiment. JCC, TX, RC, and KM analyzed the sequence read data. CKR, MB, HB, CDA (molecular biology) and SJ (IT) generated the data. JMJ provided guidance on the initial design, approved the project, and provided critical review of the project and manuscript. CAR coordinated and provided critical scientific input to the project and manuscript. JCC wrote the manuscript. All authors have approved the manuscript.

\section{Acknowledgements}

We thank David Haynor, Tomas Babak, Xinwei She, and John Lamb for suggestions, Melissa Cline for help loading tracks into the UCSC Genome Browser, and two anonymous reviewers for comments. All authors were employees of Rosetta Inpharmatics, which was a part of Merck \& Co., Inc. Merck approved release of this manuscript.

\section{Author Details}

'Rosetta Inpharmatics LLC, a wholly owned subsidiary of Merck \& Co., Inc., Seattle, Washington 98109, USA, 2Institute for Translational Oncology and Immunology (TrOn), Mainz, Germany, ${ }^{3}$ NuGEN Technologies, Inc., Seattle, Washington, USA, ${ }^{4}$ University of Washington, Seattle, Washington, USA, ${ }^{5}$ Pfizer, Inc., San Diego, California, USA, ${ }^{9}$ Merck Research Laboratories, Boston, Massachusetts, USA and ${ }^{7}$ Amgen, Inc., Seattle, Washington, USA

Received: 15 October 2009 Accepted: 16 April 2010

Published: 16 April 2010

\section{References}

1. Hirsch FR, Witta S: Biomarkers for prediction of sensitivity to EGFR inhibitors in non-small cell lung cancer. Curr Opin Oncol 2005, 17(2):118-122.

2. Wang TL, Diaz LA Jr, Romans K, Bardelli A, Saha S, Galizia G, Choti M, Donehower R, Parmigiani G, Shih le M, et al:: Digital karyotyping identifies thymidylate synthase amplification as a mechanism of resistance to 5-fluorouracil in metastatic colorectal cancer patients. Proc Natl Acad Sci USA 2004, 101(9):3089-3094.

3. Wang J, Wang W, Li R, Li Y, Tian G, Goodman L, Fan W, Zhang J, Li J, Zhang J, et al:: The diploid genome sequence of an Asian individual. Nature 2008, 456(7218):60-65.

4. Ley TJ, Mardis ER, Ding L, Fulton B, McLellan MD, Chen K, Dooling D, Dunford-Shore BH, McGrath S, Hickenbotham M, et al:: DNA sequencing of a cytogenetically normal acute myeloid leukaemia genome. Nature 2008, 456(7218):66-72.

5. Gnirke A, Melnikov A, Maguire J, Rogov P, LeProust EM, Brockman W, Fennell T, Giannoukos G, Fisher S, Russ C, et al: Solution hybrid selection with ultra-long oligonucleotides for massively parallel targeted sequencing. Nat Biotechnol 2009, 27(2):182-189.

6. Mortazavi A, Williams BA, McCue K, Schaeffer L, Wold B: Mapping and quantifying mammalian transcriptomes by RNA-Seq. Nat Methods 2008, 5(7):621-628.
7. Campbell PJ, Stephens PJ, Pleasance ED, O'Meara S, Li H, Santarius T, Stebbings LA, Leroy C, Edkins S, Hardy C, et al:: Identification of somatically acquired rearrangements in cancer using genome-wide massively parallel paired-end sequencing. Nat Genet 2008, 40(6):722-729.

8. Chiang DY, Getz G, Jaffe DB, O'Kelly MJ, Zhao X, Carter SL, Russ C, Nusbaum C, Meyerson M, Lander ES: High-resolution mapping of copynumber alterations with massively parallel sequencing. Nat Methods 2009, 6(1):99-103.

9. Alkan C, Kidd JM, Marques-Bonet T, Aksay G, Antonacci F, Hormozdiari F, Kitzman JO, Baker C, Malig M, Mutlu O, et al:: Personalized copy number and segmental duplication maps using next-generation sequencing. Nat Genet 2009, 41(10):1061-1067.

10. Yoon S, Xuan Z, Makarov V, Ye K, Sebat J: Sensitive and accurate detection of copy number variants using read depth of coverage. Genome Res 2009, 19(9):1586-1592.

11. Ruas M, Peters G: The p16INK4a/CDKN2A tumor suppressor and its relatives. Biochim Biophys Acta 1998, 1378(2):F115-177.

12. Weinberg RA: Fewer and fewer oncogenes. Cell 1982, 30(1):3-4.

13. Greenman CD, Bignell G, Butler A, Edkins S, Hinton J, Beare D, Swamy S, Santarius T, Chen L, Widaa S, et al:: PICNIC: an algorithm to predict absolute allelic copy number variation with microarray cancer data. Biostatistics 2010, 11(1):164-175.

14. Cooper GM, Zerr T, Kidd JM, Eichler EE, Nickerson DA: Systematic assessment of copy number variant detection via genome-wide SNP genotyping. Nat Genet 2008, 40(10):1199-1203.

15. Bogenhagen D, Clayton DA: The number of mitochondrial deoxyribonucleic acid genomes in mouse $L$ and human HeLa cells. Quantitative isolation of mitochondrial deoxyribonucleic acid. J Biol Chem 1974, 249(24):7991-7995.

16. Hastie ND, Dempster M, Dunlop MG, Thompson AM, Green DK, Allshire $\mathrm{RC}$ : Telomere reduction in human colorectal carcinoma and with ageing. Nature 1990, 346(6287):866-868.

17. Wang TL, Maierhofer C, Speicher MR, Lengauer C, Vogelstein B, Kinzler KW, Velculescu VE: Digital karyotyping. Proc Natl Acad Sci USA 2002, 99(25):16156-16161.

18. Leary RJ, Cummins J, Wang TL, Velculescu VE: Digital karyotyping. Nat Protoc 2007, 2(8):1973-1986.

19. Fan HC, Blumenfeld YJ, Chitkara U, Hudgins L, Quake SR: Noninvasive diagnosis of fetal aneuploidy by shotgun sequencing DNA from maternal blood. Proc Natl Acad Sci USA 2008, 105(42):16266-16271.

20. Li H, Durbin R: Fast and accurate short read alignment with BurrowsWheeler transform. Bioinformatics 2009, 25(14):1754-1760.

\section{doi: 10.1186/1471-2164-11-244}

Cite this article as: Castle et al., DNA copy number, including telomeres and mitochondria, assayed using next-generation sequencing BMC Genomics 2010, 11:244

\section{Submit your next manuscript to BioMed Centra and take full advantage of:}

- Convenient online submission

- Thorough peer review

- No space constraints or color figure charges

- Immediate publication on acceptance

- Inclusion in PubMed, CAS, Scopus and Google Scholar

- Research which is freely available for redistribution

Submit your manuscript at www.biomedcentral.com/submit
C) Biomed Central 